\title{
CURRENT CHALLENGES OF AGILE HARDWARE DEVELOPMENT: WHAT ARE STILL THE PAIN POINTS NOWADAYS?
}

\author{
Atzberger, Alexander; Paetzold, Kristin \\ Bundeswehr University Munich
}

\begin{abstract}
Originally developed for the software industry, agile development is being applied in the hardware field nowadays as well due to its benefits when having to deal with volatile, uncertain, complex and ambiguous (VUCA) conditions. However, certain complications arise when applying the concept of agility in the hardware. In this publication, based on the challenges identified by Ovesen in 2012, the current challenges of agile development for hardware are gathered using an embedded design approach, ensuring its actuality based on the latest surveys and empirical data. The current state regarding the challenges is displayed and its interrelations as well as their advancements compared to seven years ago is discussed. Moreover, an attempt to explain the difficulties of applying agile development is given by a hardware-related complexity model.
\end{abstract}

Keywords: Agile hardware development, Research methodologies and methods, Design methodology, New product development

\section{Contact:}

Atzberger, Alexander

Bundeswehr University Munich

Institute for Technical Product Development

Germany

alexander.atzberger@unibw.de

Cite this article: Atzberger, A., Paetzold, K. (2019) 'Current Challenges of Agile Hardware Development: What are Still the Pain Points Nowadays?', in Proceedings of the 22nd International Conference on Engineering Design (ICED19), Delft, The Netherlands, 5-8 August 2019. DOI:10.1017/dsi.2019.227 


\section{INTRODUCTION}

The definition of what is considered to be agility nowadays was constituted back in 2001, when 17 software engineers were gathering to agree upon a different working philosophy, fitting the needs and circumstances of their branch at that time. With the Manifesto being the foundation of agile development, the cornerstone has been set and agility has been on the rise ever since, resulting in the upcoming of several agile methods over the years. Yet, the fundamental grasp all agile methods share is their compliance to the 'Agile Manifesto'. Most popular methods such as Scrum (originally already introduced in 1995), Kanban, eXtreme Programming (XP) or Crystal are widely used in the software industry today. They consist of agile principles (which they partially share) defining the working mechanisms which are concretized by agile practices. Figure 1 displays the basic structure of agile development in a generic way, with the 'Agile Manifesto' being the guiding methodology, the resulting methods and its subsequent breakdown into principles and practices (Schmidt et al., 2018a).

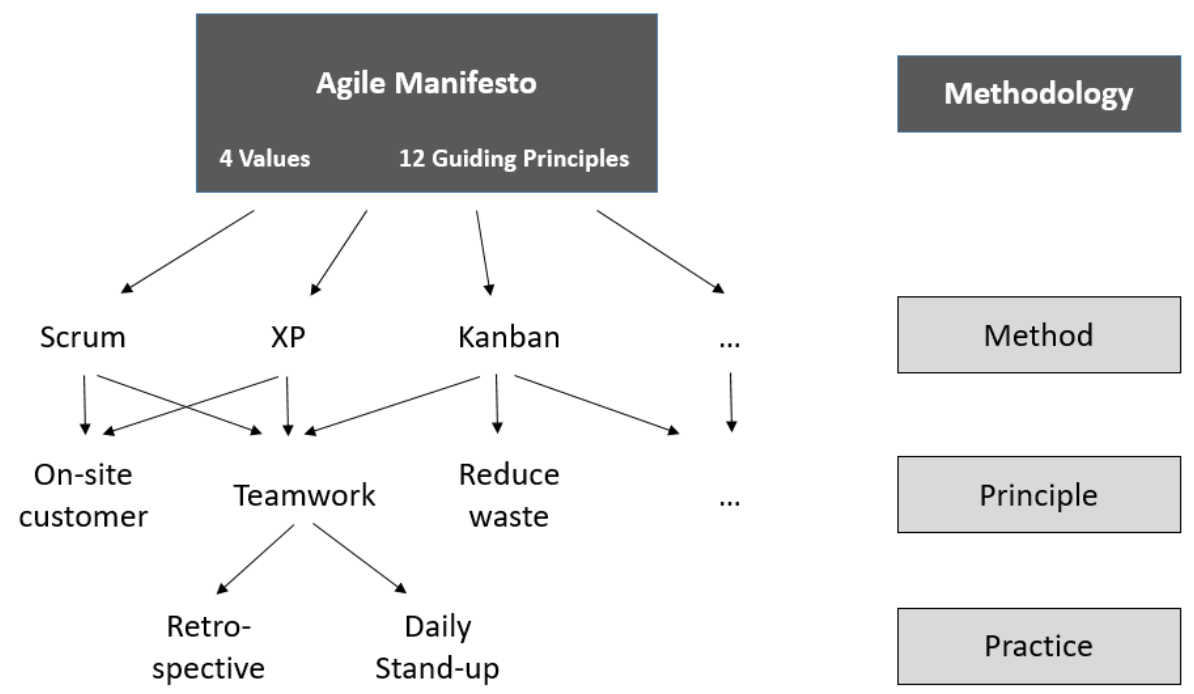

Figure 1. Outline of the structure of agile development according to (Schmidt et al., 2018a)

With an agile method consisting of a set of principles and practices, its aim is to enhance the modus operandi in a specific subdomain by being able to cope with the affiliated defiances. Thus, these methods are context-dependent, addressing challenges in and by the respective environment. Scrum, being the most popular agile method nowadays, has a rather work-coordinating character (supporting the handling and management of the project). XP on the other hand, which was popular especially in the early days of agile development, has a rather product-design focus. Over time, some of the methods have partially been refined and several combinations of agile methods such as ScrumBan, Lean Kanban or Xanpan were established, combining several principles and practices from different methods (Kelly, 2015). These advancements show, that it is necessary to align agile methods contextspecific and also to the respective structure of the company applied to. With the success of those methods under VUCA conditions, the shift to other fields has been undertaken, spreading also into the field of hardware development. In this field, however, well-established traditional development approaches such as the Waterfall- or Stage-Gate model are prevailing (Cooper, 2011; VDI, 2004). Those classical approaches are highly effective and efficient under stable conditions; however, they are not able to cope with ever-changing boundary conditions resulting in frequent requirement changes very well. Due to the complexity of both products as well as organizations, yet the necessity to be competitive in under VUCA conditions, agile methods are introduced in pilot projects. Several companies developing hardware have already implemented agile methods, Scrum in specific (Komus et al., 2018). According to consultancies in the field of hardware $\mathrm{R} \& \mathrm{D}$, these turn out to be quite successful (Schröder and Schrofner, 2015). Proposed agile concepts are introduced and adapted to company-specific environments in order to be able to work accordingly. (Ovesen, 2012) has summarized a broad set of challenges associated with the application of agile methods, Scrum in specific, in seven Danish companies at that time. Dating back seven years from now, the aim of this 
publication is to review the challenges identified by Ovesen and compare them to the current state of agile hardware development. Thus, the following the following research questions arise:

Are the challenges associated with agile hardware development, as stated by Ovesen in 2012, still valid? How can the difficulty of transferring agile development into the field of hardware be characterized?

The aim of this research is to give a holistic overview over the challenges that are still associated with the agile development of physical products, as of 2018. By doing so, both academia and industry will be able to focus on the core issues and thus able to determine ways and measures on how to overcome them.

\section{STATE OF THE ART}

The terms "hardware development" and "development of physical products" are used analogously throughout this publication. These terms refer to products, which do not consist of software solemnly and thus bear a physical nature, incorporating elements of the hardware domain combined with electronic and software domain. The results are mechatronic or cyber-physical products (Schmidt et al., 2018 b). With the interplay of those three domains into one product, several challenges regarding their development under VUCA conditions arise. Thus, the need for measures on how to cope with these difficulties in the field of hardware arise accordingly. With the successful implementation of agile methods in the field of software, meanwhile being state of the art in this domain (VersionOne, 2018), the most popular method by far is considered to be Scrum (Link, 2014). According to the latest version of the Scrum Guide (Schwaber and Sutherland, 2017), the authors of the Scrum framework claim that it is applicable in the hardware domain now as well. Based on that statement, the challenges associated with the adoption of agile methods in physical product development are examined in more detail.

(Ovesen, 2012) has analysed seven Danish companies developing physical products by the use of Scrum back in 2012 and issued a broad set of challenges. Between the issue of both sources is a time span of five years. The challenges identified by Ovesen will be taken as a basis for the further analysis. Those were clustered into five superordinate groups, naming constraints of physicality, paradigm perplexity, designer's dissent, team distribution dilemma and education and maturation.

In contrast to virtual products, physical products faces the so-called 'constraints of physicality'. These can be summarized as the sum of challenges that occur due to the physical nature of hardware products, thus they are unique to the hardware domain. The building and testing of a hardware prototype is much more time-consuming compared to the writing and compiling of a software. In this respect, e.g. the frequent generation of prototypes (increments) in short iterations is still considered one major challenge (Bahlow et al., 2013; Ovesen and Dowlen, 2012). Alongside, the challenge of long production / delivery times of certain hardware components comes into play (Böhmer et al., 2017). However, the constant delivery of working increments is essential for agile development to test uncertainties and receive reliable feedback (Böhmer et al., 2015). In this respect, the breakdown of the product increment into small tasks is considered challenging (Ovesen and Dowlen, 2012). The term 'paradigm perplexity' refers to the difficulty of "transferring [...] a radically new development paradigm into a traditional development environment", "integrating two contradicting process models with conflicting values" (Ovesen, 2012). In this context, the introduction of agile methods as well as the further rollout inside the company, nowadays referred to as 'scaling' is becoming a large issue (Eklund and Berger, 2017; Gregory et al., 2015). In this respect, agile scaling frameworks such as SAFe or LeSS are gaining attention (Brenner and Wunder, 2015; Larman and Vodde, 2016). The term 'designer's dissent' refers to willingness of applying and acting according to agile methods. In this respect, the topic of cooperation (Bahlow et al., 2013; Ovesen and Dowlen, 2012) is an issue. Furthermore, the "application of agile methods in sectors where application experiences are rare" are also challenging (Gregory et al., 2015). Linked to these, the "change in attitude", leaving the old habits behind, goes along with it (Bahlow et al., 2013; Gregory et al., 2015). The "team distribution dilemma' can be explained as the inability to work co-located, as initially outlined by the Manifesto. Due to this, challenges regarding the coordination originate, resulting in a high degree of communication needed (Paasivaara et al., 2009). In this context, the extensive need of knowledge sharing and the constant information exchange is an impediment (Conforto et al., 2014). The subject of 'education and maturation' deals with the initial introduction of agility, its common understanding and acceptance in the first place, and the maturation of agility as a concept in the second place, which is a long-running process (Hoda et al., 2018; Ovesen, 2012). Agility has to be understood as a 
different way of working, meaning that the company culture has to change accordingly in order to succeed (Dikert et al., 2016).

\section{RESEARCH DESIGN}

For this investigation, an embedded design approach has been used. Based on the data obtained from literature, a secondary data analysis has been conducted. The latest surveys determining the status quo of agile development in general (VersionOne, 2018), as well as focused on agile and traditional methods for hardware (Komus et al., 2018) and agility in the hardware domain (Schmidt et al., 2018a; We.Conect, 2018) have been analysed regarding the current challenges of agile development of physical products. The relevant data has been extracted and the dataset has been updated, building the foundation to be validated in the further course. In order to guarantee the actuality of the findings, the results were discussed with experienced practitioners during two workshops. These qualitative findings helped to (a) gain a deeper understanding about how and why these challenges occur and (b) to validate the findings from the dataset. This first version has been discussed with eleven experts from academia and practitioners from the industry in a two-day workshop focusing on the latest advancements in agile hardware development (Workshop 1). The practitioners are experts for agile hardware development in their companies (developing mechatronic products) and thus have deep understanding of the topic of agility and the challenges associated with it. In three iterations, the challenges were discussed and elaborated following the double diamond practice from Design Thinking. Based on the insights of that workshop, the dataset was refined and additional information was added. In the next step, the results were presented at the Agile PEP Minds ' 18 conference in Berlin in November 2018. In a world café session, where a topic is presented by the session host and then discussed with the participants, the results were presented consecutively to five groups of practitioners (10 - 12 practitioners per group), each session lasting 30 minutes. Based on the first-hand experiences of the practitioners, the dataset has been aligned again. Additionally, several valuable insights from practitioners in one-on-one talks sharpened the characteristics of the results (Workshop 2). After having incorporated these findings, the final version of the investigation has been completed. The results are intended to give a holistic overview over the current challenges associated with agile hardware development, as of late 2018, and to emphasize on the main issue that still hamper the its application.

\section{FINDINGS}

The results of this investigation regarding the current challenges associated with agile hardware development have been elaborated and clustered. Overall, the results are condensed into the following four main categories: Constraints of physicality, Mindset, Scaling and Team Distribution. This categorisation has been made by the author based on the results gathered. The key aspects related to each category are summarized in Table 1 . The impact of the respective challenge and the interrelation between those are elaborated in the subsequent description.

Besides the specific challenge and the category related to, the sources from surveys are marked as follows: (VersionOne, 2018) - S1, (Komus et al., 2018) - S2, (Schmidt et al., 2018a) - S3, (We.Conect, 2018) - S4. The validation steps are marked (Workshop 1 - V1, Workshop 2 - V2) accordingly.

Constraints of physicality (CoP): The challenges related to the constraints of physicality have to be split into different sections. Given the iterative and incremental nature of agile development, the greatest hindrance is (1) to realize potentially shippable increments in one iteration. Moreover, even the creation of a prototype (let alone deliverability) to test uncertainties is hardly achievable in some industrial branches. In this context, (2) the technical feasibility to produce prototypes in a short time span is one concern. One aspect that goes along with that issue is (3) the inability to break down the product into modules to be tested. Given the complexity of mechatronic systems, the interplay between hardware, software and electronics needs to be taken into account. In this respect, (4) external dependencies come into play, since several components are commonly not produced in-house, which is one major cause of the inability to frequently produce prototypes. Ranging from electrical parts such as chips or circuit boards to mechanical parts to be manufactured using special processes, time delays due to the mechanical production and supply occur. Following the delivery of those components, they still have to be implemented and tested. However, several components cannot be produced with stock- 
material, leading to dependencies regarding (5) the production of tools, which can be a very timeconsuming matter. In addition to that, the testing of components for specific, high-tech applications cannot be completed within short cycle times. Especially in these branches (6) documentation and certifications, when the interaction with humans can lead to a life-threating issue in case of failure, are also time-consuming and have to be performed by testing organizations, leading to additional external dependencies. Due to the incorporation of three domains into one mechatronic product, (7) the specialisation of the individual and thus the availability to several development teams is also challenging. Given the complexity of the product, (8) the synchronisation of the domains and the coordination of the interfaces are defiant.

Given that, (9) the frequent stakeholder feedback is another difficulty, since people in the field of hardware (used to work according to classical process models) commonly do not see the benefit in giving feedback on some hypothetical, non-functional prototype. In their opinion, a prototype is something "close to being ready", fulfilling functional requirements such as a MVP.

Table 1. Current challenges and the respective sources

\begin{tabular}{|c|c|c|c|}
\hline No. & CHALLENGE & CATEGORY & SOURCE \\
\hline 1 & Realization of potentially shippable increments & \multirow{9}{*}{$\mathrm{CoP}$} & $\mathrm{S} 2, \mathrm{~S} 3, \mathrm{~S} 4, \mathrm{~V} 1, \mathrm{~V} 2$ \\
\hline 2 & Technical feasibility to produce prototypes & & $\mathrm{S} 2, \mathrm{~S} 3, \mathrm{~V} 1, \mathrm{~V} 2$ \\
\hline 3 & Inability to break down the product into modules & & $\mathrm{S} 2, \mathrm{~S} 3, \mathrm{~V} 1, \mathrm{~V} 2$ \\
\hline 4 & External dependencies & & $\mathrm{S} 3, \mathrm{~S} 4, \mathrm{~V} 1, \mathrm{~V} 2$ \\
\hline 5 & Production of tools & & $\mathrm{S} 3, \mathrm{~S} 4, \mathrm{~V} 1, \mathrm{~V} 2$ \\
\hline 6 & Documentation and certifications & & $\mathrm{S} 2, \mathrm{~S} 3, \mathrm{~S} 4, \mathrm{~V} 2$ \\
\hline 7 & Specialisation of the individual & & $\mathrm{S} 2, \mathrm{~S} 3, \mathrm{~V} 1, \mathrm{~V} 2$ \\
\hline 8 & Synchronisation of the domains & & $\mathrm{S} 2, \mathrm{~S} 3, \mathrm{~V} 1, \mathrm{~V} 2$ \\
\hline 9 & Frequent stakeholder feedback & & $\mathrm{S} 1, \mathrm{~S} 3, \mathrm{~V} 1, \mathrm{~V} 2$ \\
\hline 10 & Establishing an agile mindset & \multirow{8}{*}{ Mindset } & $\mathrm{S} 1, \mathrm{~S} 2, \mathrm{~S} 3, \mathrm{~S} 4, \mathrm{~V} 1, \mathrm{~V} 2$ \\
\hline 11 & Proper education and training & & $\mathrm{S} 1, \mathrm{~S} 2, \mathrm{~S} 3, \mathrm{~S} 4, \mathrm{~V} 1, \mathrm{~V} 2$ \\
\hline 12 & Incorporating an agile team into a classical company structure & & $\mathrm{S} 2, \mathrm{~S} 3, \mathrm{~S} 4, \mathrm{~V} 2$ \\
\hline 13 & "Prince problem" & & $\mathrm{S} 2, \mathrm{~V} 1, \mathrm{~V} 2$ \\
\hline 14 & Commitment of the top management & & $\mathrm{S} 1, \mathrm{~S} 2, \mathrm{~S} 3, \mathrm{~V} 1, \mathrm{~V} 2$ \\
\hline 15 & Commitment of the middle management & & $\mathrm{S} 2, \mathrm{~S} 3, \mathrm{~S} 4, \mathrm{~V} 1, \mathrm{~V} 2$ \\
\hline 16 & Multi-project management & & $\mathrm{S} 2, \mathrm{~S} 3, \mathrm{~S} 4, \mathrm{~V} 1, \mathrm{~V} 2$ \\
\hline 17 & Internal process models & & $\mathrm{S} 2, \mathrm{~S} 3, \mathrm{~S} 4, \mathrm{~V} 1$ \\
\hline 18 & Transfer of (methodological) knowledge & \multirow{5}{*}{ Scaling } & $\mathrm{S} 1, \mathrm{~S} 2, \mathrm{~S} 3, \mathrm{~V} 1, \mathrm{~V} 2$ \\
\hline 19 & Structure of the company & & $\mathrm{S} 1, \mathrm{~S} 2, \mathrm{~S} 3, \mathrm{~S} 4, \mathrm{~V} 1, \mathrm{~V} 2$ \\
\hline 20 & Silo mentality & & $\mathrm{S} 2, \mathrm{~S} 3, \mathrm{~V} 1$ \\
\hline 21 & Mindset change of the organization & & $\mathrm{S} 1, \mathrm{~S} 2, \mathrm{~S} 3, \mathrm{~V} 1, \mathrm{~V} 2$ \\
\hline 22 & Adaptation to the company-specific values & & $\mathrm{S} 1, \mathrm{~S} 2, \mathrm{~S} 3, \mathrm{~S} 4, \mathrm{~V} 1, \mathrm{~V} 2$ \\
\hline 23 & Communication of distributed teams & \multirow{3}{*}{$\begin{array}{c}\text { Team } \\
\text { Distribution }\end{array}$} & $\mathrm{S} 2, \mathrm{~S} 4, \mathrm{~V} 1, \mathrm{~V} 2$ \\
\hline 24 & Usage of communication tools & & $\mathrm{S} 2, \mathrm{~V} 2$ \\
\hline 25 & Ethical and cultural differences & & $\mathrm{V} 1, \mathrm{~V} 2$ \\
\hline
\end{tabular}

Mindset: Another major hindrance is the topic, which is referred to as mindset here. In this publication, this term shall serve as superordinate for the understanding of agility by the individual on the one hand, and the company (as a body of individuals) and its associated company culture on the other hand.

Starting with the individual, one major challenge is (10) to establish an agile mindset in the heads of the individuals. Driven by the current hype about agility in the hardware (Schmidt et al., 2018a), people tend to misunderstand what agility is actually about. Especially when introducing the concept of agility to an existing team that has worked in a plan-driven manner before, the rethinking on how to accomplish the same task as before with a different approach is challenging. It has to be understood that working in an agile manner is a change in terms of collaboration, granting more freedom to the individual, yet also more responsibility to every team member. (11) Proper education and training, and the willingness to accept this new working style, are essential to be understood by everyone within the team. The application of practices 
such as daily stand-up meetings and retrospectives is intended to improve transparency and highlight problems, so that they can be discussed and overcome within the team. If this important aspect of an openerror culture is not communicated and acted out properly, the individuals refuse to speak about the challenges and mistakes in fear of admonition. If this is the case, working in an agile manner is doomed to fail, resulting in frustration of the individual and resistance to work according to agile methods.

Shifting to the aspect of company culture, the traditional way of thinking has to be overcome. When introducing agility and acting it out in a pilot project inside a company, several aspects have to considered that can lead to challenges. Introducing a topic such as agility into a company has to be understood as a process. Especially inside a mature company that looks back on a longer history, traditions and routines predominate. (12) Incorporating an agile team into a classical company structure often leads to issues on the interfaces with surrounding departments. Since development teams are part of an overall company structure, the circumstances have to be adapted for the team to act out in an agile manner. Especially in industrial sectors that are considered conservative, establishing an agile team inside a company's R\&D department is considered very challenging. Let alone the dependencies regarding the willingness to give feedback to increments by both external and internal stakeholders, also the internal structures and processes hinder the team to work in an agile manner. In such companies, the success of agility strongly depends on the commitment of the top management and their willingness to align prevailing routines and habitual procedures inside the company structure. The success of such pilot projects is determining for the further pursuit of agile development.

Hierarchical structures have to be adapted by the company, resulting in a flat hierarchy and thus a loss of power in the (senior) management. This issue is referred to as the (13) "prince problem", resulting in a loss of responsibility of the managers and thus prestige. In classical structures, the (project) managers are in charge of decision-making, directing orders to the team members, whereas in an agile team the role of the "team leader" is more of an enabler, supporting and empowering the people to do whatever is necessary in order to achieve the overall task, granting freedom and power to the team members. Such a shift in the understanding of one's role has to be accepted by both the (original) team members and the team leader in order to work accordingly. Taking it one step further, the application of agile methods in the company structure as a whole needs to be approved, resulting in (14) commitment of the top management as well as (15) the middle management. If a team is lacking this commitment, working in an agile way cannot be successful, since the team needs to be able to take decisions and have the freedom to act accordingly in order to proceed with its work. Introducing agility inside a company bottom-up can only be successful, if the top management supports this approach. Additionally, (16) multi-project management should be avoided for the team to be able to focus on the current project and (17) internal process models also need to be aligned accordingly. If the traditional culture of the company is not actively forced to change and the topic of agility is not supported by the top management, "culture eats strategy for breakfast" (V2), resulting in frustration and resistance in the heads of the people, leaving scorched earth.

Scaling: When having completed the successful implementation of agility in a pilot project, rolling out agility in several projects, referred to as scaling, is the next step in the agile transformation. In this respect, one challenge is (18) to transfer the (methodological) knowledge gathered in and with the application of agile methods to the subsequent teams. The members of the pilot teams had to undergo a process of learning on what went well, on how to overcome team-internal challenges or problems and they experienced the benefit of frequent communication. To transfer this knowledge without active support of one of the original team members is leading to the frustration of the individual, as mentioned above. Along with that, (19) the structure of the company has to be aligned accordingly, as already mentioned in the paragraph 'company culture'. Changes in the project organization follow due to an adaption of the team structures. This is inevitable in order to circumvent (20) the issue of the silo mentality, with each department rather focussing on their respective tasks than the overall project. Having multi-disciplinary teams with members of each domain counteracts this effect. Yet, when running multiple teams to cope with a greater complexity of a project, more interfaces to other departments exist, such as the purchasing or controlling department. Additionally, with rising product complexity the challenge of dependencies in terms of external compliance come along. Moreover, (21) the mindset change of the organization as a whole and its acceptance of agile scaling frameworks such as LeSS, Scrum of Scrum, Nexus or SAFe is currently one of the greatest challenges associated with scaling. (22) The adaptation to the company-specific values has to be undertaken, however, in many 
cases, these framework do not sufficiently fit to the existing, prevailing structure of the companies. In this respect, the aspect of senior management commitment and middle management power loss recurs since the hierarchy will be flattening out over time throughout the transformation process.

Distribution of teams: With many companies having subsidiaries, the topic of collaboration of teams throughout different locations is a very important aspect from the viewpoint of companies. Especially the challenge of (23) communication of distributed teams is one major aspect, which is an unresolved issue. Especially when team members from different domains are split area-wise, the dislocation hinders the team members to actively engage in conversations in order to solve technical issues. In this respect, the question on how to perform e.g. daily stand-ups is unclear, when developers from different time zones have to collaborate, meaning people from USA have to collaborate with team members from Europa or Asia. Besides that, challenges on (24) which communication tools to use in order to thoroughly understand the team members comes into play. This aspect is underestimated when people from different countries have to cooperate and language barriers (due to the non-nativity of the language) hamper the collaboration of the individual team members. Along with that, also (25) ethical and cultural differences are considered a challenge, since open criticism and an open-error culture is not tolerated by every nationality. However, when transparency is traded for the sake of a harmonic atmosphere, the issues are predetermined to backfire.

\section{DIscussion}

\subsection{Evolution of the challenges of AHD in comparison to 2012}

The findings show, that most of the initial challenges identified by Ovesen are still valid nowadays. The alignment regarding the categories has been applied because the superordinate term of 'mindset' covers all of the aforementioned aspects of Ovesen. Yet, the general structure is in close accordance to Ovesen, since it was considered most feasible. Where necessary, terms have been adapted and added, based on the results of the research.

Back in 2012, Ovesen has been one of the first to investigate the application of an agile method in the context of physical product development. Since the topic of 'agility' has been around for approx. ten years back then, especially in the software branch, the agile development of physical products was quite uncommon back then and hardly subject to research. Due to this, the practitioners did not have year-long experience with the application of agile methods and were rather unexperienced. The lack of maturity could also be seen that aspects like the team morale and the task breakdown were seen as major problem areas. Thus, certain challenges were not present at that time because they have not been experienced yet, such as the topic of scaling or the 'prince problem'. An advancement of the practitioners in the field of agile hardware development is therefore recognizable in the upcoming of new challenges, e.g. by shifting to implement the concept of agility throughout the whole company, undertaking an agile transition. This is, of course, driven by the current hype around agile hardware development. In this respect, several challenges are still apparent, while others have transformed and evolved by the further adoption of agile methods within the teams, with the teams, after initial euphoria, tend to experience the limits and get disillusioned, before they move on to the slope of enlightenment and find suitable approaches to tackle their challenges (Schmidt et al., 2018a). In an intermediate step in the generalisation, Ovesen split the topics in development challenges and organisational challenges, which is very simply, but meaningful way of differentiation, which has also been chosen in the latest study regarding the current state of agile hardware development (Schmidt et al., 2019). Following that step, Ovesen has defined the five superordinate groups, as explained in the State of the Art. When analysing the wording of these five groups by Ovesen and the four terms chosen in this contribution, a shift towards a generalization is recognizable, which underpins the maturation of the topic from 2012 up to today.

Ovesen has stated "the constraints of physicality to be the major critical hindrance to fully comply with Scrum", as of 2012. Based on the findings, the constraints of physicality are still a major challenge when adopting agile methods in the field of hardware. Due to the fact that more and more companies are striving to implement agile development into their hardware $R \& D$, also the variety in challenges is rising accordingly. The core issue in this respect are either lengthy tooling or manufacturing process or external dependencies. For hardware products that are rather simple in its architecture, ways and means on how to develop them in an agile manner has already been achieved. (Schmidt et al., 2017) have developed the 
Adapted Media Richness Theory, which displays which kind of prototype is meaningful in order to test specific uncertainties. The knowledge base can be extended faster when choosing an appropriate kind of prototype instead of building a high-fidelity mock-up. The topic of the mindset however is emerging resp. apparent nowadays. Driven by the well-suited applicability of agile methods in the software domain, the application of agility for hardware is being hyped, resulting in unmet expectations due to insufficient means on how to cope with the variety of the aforementioned challenges. (Schmidt et al., $2018 \mathrm{~b}$ ) have analyzed the current hype of agile hardware development, displaying the progress in Gartner's Hype cycle. The results show, that introduction of agile methods into the field of hardware is approximately ten years behind the agile software development.

If the capabilities, limitations and possible adaptions of agile methods is not communicated properly, frustration and refusal are the result. This plays especially an important role when agile development is rolled-out in a company in order to scale. The insufficient approaches regarding agile hardware scaling frameworks adds to that issue, since agility was actually not intended to be used in large groups resp. teams. The same takes effect for the matter of team distribution, with collaboration being aggravated due to its non-intentional use. Especially in those fields, further research is necessary. Overall, it can be summarized that the problem space regarding the challenges has increased. Especially due to the success of Scrum in the software domain, it is also the most common method (with adaptions) to hardware as well (Komus et al., 2018). This is a plus in terms of the experience gained whilst working with agile methods, yet the simple transferability to the hardware domain is hardly achievable, due to the intricacy of the interplay of the three domains mechanics, electronics and informatics. Consulting experts in the field of agile development, being either external consultants or internal Agile Coaches is definitely one reasonable measure, since the methodological knowledge has to be indoctrinated into the company with context-specific adaptions made. Due to this, a change in the company culture can be initiated easier as if those measures are not taken. In order to support companies in maturing in the application of agile methods and to overcome the "trough of disillusionment", which is yet to come according to the Hype cycle, academia has to provide respective solution approaches.

\subsection{Difficulty of transferring to agile hardware development}

Since experienced practitioners from the software industry have originally developed the "Agile Manifesto' for the software industry, it does not cover aspects of hardware development in any way. Yet, the core values and guiding principles can partially be applied to the hardware domain per se as well. The transferability of the Manifesto to the hardware domain is also gaining a broader understanding according to the latest survey data by (Schmidt et al., 2018a), rating the transferability $3,9 / 5(\mathrm{n}=91)$. Yet, the hardware-specific constraints listed above have not been addressed in a comprehensive method, particularly designed for hardware, yet. However, as stated in the findings, approaches and guidelines on how to tackle the challenges associated with e.g. the constraints of physicality are necessary, since the current popular methods cannot cope with those adequately. Therefore, the challenge of addressing the field of hardware development is elaborated in more detail. Mechatronic and cyber-physical products consist of elements of software, electronics and hardware (tangible, physical components). Hence, the complexity in the field of hardware development has a much broader range than in the software. Figure 2 is an attempt to explain this difficulty by displaying the boundary conditions concerning the development environment and thus, the 'sweet spot' for agile hardware development in three dimensions. In this hardware-related complexity illustration, the ordinate represents the complexity range in terms of the product architecture, ranging from low (few components) to very high (several components from all domains with a high level of specialisation). The abscissa represents the company size, ranging from small (i.e. small business) to very large (i.e. multicorporate enterprise with several subsidiaries in different countries). The applicate represents the size of the project team developing the respective product, ranging from one small team to several teams / large team sizes. The aim of this characterization is to illustrate the boundary conditions that are related to the field of hardware in specific. In contrast to software development, the characteristics regarding the hardware-related complexity of agile hardware development are versatile. Taking a closer look at the dimension of product architecture, it needs to be interpreted as the interplay of the three domains in order to come up with a highly competitive, integrated product. 


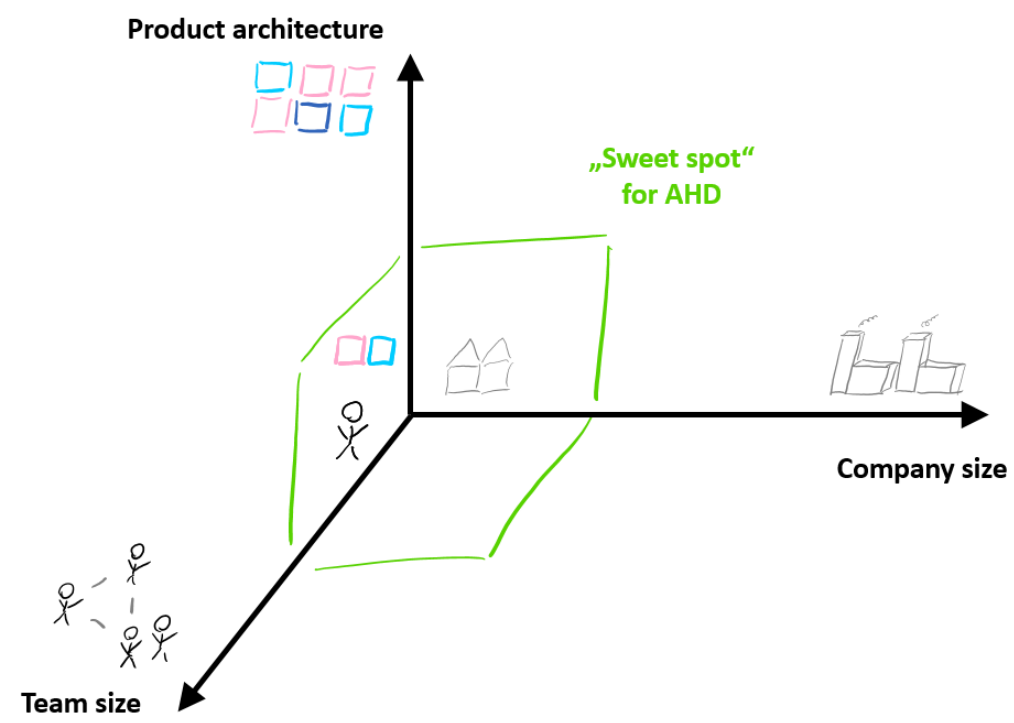

Figure 2. Scheme of hardware-related context conditions for agile hardware development

This dimension incorporates many facets and is therefore the most diversified dimension, which needs to be apportioned specifically for the context of agile hardware development. One aspect of this dimension is that depending on the extent of the product to be designed, its composition can vary broadly, increasing up to hundreds of components into one single product (e.g. cars or airplanes). Regarding the size of the company, the environment in which the product is being designed can vary a lot. Working in a small company developing only a few products, holding a "start-up" like atmosphere with a lot of freedom granted to the team differs significantly to a large enterprise, incorporating multiple subsidiaries, with rigid company structures, operational constraints and thus distinctively less freedom. The team size can also differ a lot, from one single co-located team to multiple teams, across different countries. In addition, the composition of the team can vary a lot, yet the team's composition has a significant impact on the product's architecture since it is directly linked, as stated in Conway's law.

The four clusters CoP, mindset, scaling and team distribution can be found in this illustration. The CoP refer to the dimension of product architecture, whereas the mindset is to be found in both the company and team (size) dimensions. Scaling is a topic of the company size, whereas the team distribution refers to the team size-axis. The "sweet spot" for agile development in general, and when applied in the hardware, is indicated by the green area, being the field of 'greenfield innovation', since the constraints of each dimension are passable. Yet, highly sophisticated and therefore complex products are predominantly developed by large-size companies due to the necessity of incorporating different domains. However, as explained previously, this paralyzes the respective development due to guidelines, regulation and company-specific standards.

\section{CONCLUSIONS}

The aim of this publication was to display the current status on the challenges associated with agile hardware development, compared against the findings from seven years ago. Based on the challenges as of 2012 by Ovesen, the latest surveys on this topic have been analysed, the dataset has been updated and validated throughout two iterations with experts from both academia and industry. The findings display a change in challenges to the superordinate term referred to as mindset. Also, the constraints of physicality (CoP) still play a major role in the agile development of physical products. An attempt to describe the hardware-related complexity was conducted by incorporating the findings into a generic representation. One important aspect that helps to explain the inappropriateness of the current methods are the detaching requirements, which the Agile Manifesto has originally not been designed for. The superordinate challenges "scaling" and "team distribution" result from the companies' boundary conditions, yet the guiding principles of the Manifesto actually propose the opposite - one small and co-located team. With those deviations from the original Manifesto at hand, an alignment of the current methods is necessary. Adaptations to the field of hardware development are inevitable, therefore, principles and practices are necessary for the companies to circumvent the hardware-related issues, especially the constraints of physicality. Yet the concept of agility can also be applied to other fields. 


\section{REFERENCES}

Bahlow, J., Janning, J., Jebenstreit, H. and Kullmann, G. (2013), "Der Einführungsprozess", in Kullmann, G., Longmuß, J., Bullinger, A. and Spanner-Ulmer, B. (Eds.), Agiles Projektmanagement in Der Praxis, aw\&I Wissenschaft und Praxis, Chemnitz, pp. 36-45.

Böhmer, A., Beckmann, A. and Lindemann, U. (2015), "Open innovation ecosystem - makerspaces within an agile innovation process", in Huizingh, E., Conn, S., Bitran, I. (Ed.), Proceedings of the ISPIM Innovation Summit: Changing the Innovation Landscape, The International Society for Innovation Management, Brisbane, pp. 1-11.

Böhmer, A.I., Hugger, P. and Lindemann, U. (2017), "Scrum within hardware development insights of the application of scrum for the development of a passive exoskeleton", 2017 International Conference on Engineering, Technology and Innovation: Engineering, Technology and Innovation Management Beyond 2020: New Challenges, New Approaches, ICE/ITMC 2017 - Proceedings, pp. 790-798.

Brenner, R. and Wunder, S. (2015), "Scaled agile framework: Presentation and real world example”, 2015 IEEE Eighth International Conference on Software Testing, Verification and Validation Workshops (ICSTW), IEEE, pp. 1-2.

Conforto, E., Salum, F., Amaral, D., da Silva, S.L. and de Almeida, L.F.M. (2014), “Can agile project managemet be adopted by industries other than software development?", Project Management Journal, Vol. 45 No. 3, pp. 21-34.

Cooper, R.G. (2011), Winning at New Products: Creating Value Through Innovation, Basic Books, New York.

Dikert, K., Paasivaara, M. and Lassenius, C. (2016), "Challenges and success factors for large-scale agile transformations: A systematic literature review”, Journal of Systems and Software, Elsevier Inc., Vol. 119, pp. 87-108.

Eklund, U. and Berger, C. (2017), "Scaling agile development in mechatronic organizations - A comparative case study", Proceedings - 2017 IEEE/ACM 39th International Conference on Software Engineering: Software Engineering in Practice Track, ICSE-SEIP 2017, pp. 173-182.

Gregory, P., Barroca, L., Taylor, K., Salah, D. and Sharp, H. (2015), “Agile challenges in practice: A thematic analysis", Lecture Notes in Business Information Processing, Vol. 212, pp. 64-80.

Hoda, R., Salleh, N. and Grundy, J. (2018), "The rise and evolution of agile software development", IEEE Software, IEEE, Vol. 35 No. 5, pp. 58-63.

Kelly, A. (2015), Xanpan: Team Centric Agile Software Development, Software Strategy Ltd.

Komus, A., Kuberg, M., Erretkamps, H., Koch, C.-P., Böven, E., Fries, J. and Hermen, P., et al. (2018), Studie Status Quo PEP - 2018, Koblenz.

Larman, C. and Vodde, B. (2016), Lage-Scale Scrum: More with LeSS, Addison-Wesley Professional.

Link, P. (2014), “Agile Methoden im Produkt-Lifecycle-Prozess - Mit agilen Methoden die Komplexität im Innovationsprozess handhaben", Komplexitätsmanagement in Unternehmen, Springer Fachmedien Wiesbaden, Wiesbaden, pp. 65-92.

Ovesen, N. (2012), The Challenges of Becoming Agile, PhD Thesis, Aalborg University.

Ovesen, N. and Dowlen, C. (2012), "The challenges of becoming agile - experiences from new product development in industry and design education", 14th International Conference on Engineering and Product Design Education: Design Education for Future Wellbeing, Antwerp, pp. 9-14.

Paasivaara, M., Durasiewicz, S. and Lassenius, C. (2009), "Using scrum in distributed agile development: a multiple case study", Proceedings - 2009 4th IEEE International Conference on Global Software Engineering, ICGSE 2009, pp. 195-204.

Schmidt, T.S., Atzberger, A., Gerling, C., Schrof, J., Weiss, S. and Paetzold, K. (2019), Agile Development of Physical Products: An Empirical Study about Potentials, Transition and Applicability, University of the Ger man Federal Armed Forces, Munich.

Schmidt, T.S., Böhmer, A.I., Wallisch, A., Paetzold, K. and Lindemann, U. (2017), "Media richness theory in agile development: Choosing appropriate kinds of prototypes to obtain reliable feedback", 23th International ICE Conference on Engineering, Technology and Innovation (ICE'17), Madeira, Portugal.

Schmidt, T.S., Weiss, S. and Paetzold, K. (2018a), Agile Development of Physical Products: An Empirical Study about Motivations, Potentials and Applicability, University of the German Federal Armed Forces, Munich.

Schmidt, T.S., Weiss, S. and Paetzold, K. (2018b), "Expected vs. real effects of agile development of physical products: Apportioning the hype", International DESIGN Conference 2018, Dubrovnik, pp. 2121-2132.

Schröder, A. and Schrofner, A. (2015), "Hochleistung ist crossfunktional", Der F\&E Manager - Für Mehr Effizienz in Der Innovation, pp. 6-13.

Schwaber, K. and Sutherland, J. (2017), “Scrum guide 2017”, No. November, pp. 6-17.

VDI. (2004), "VDI 2206 - Entwicklungsmethodik für Mechatronische Systeme”, VDI Handbuch, Berlin.

VersionOne. (2018), 12th Annual State of Agile Report.

We.Conect. (2018), Agile PEP Minds Survey 2018, Berlin. 